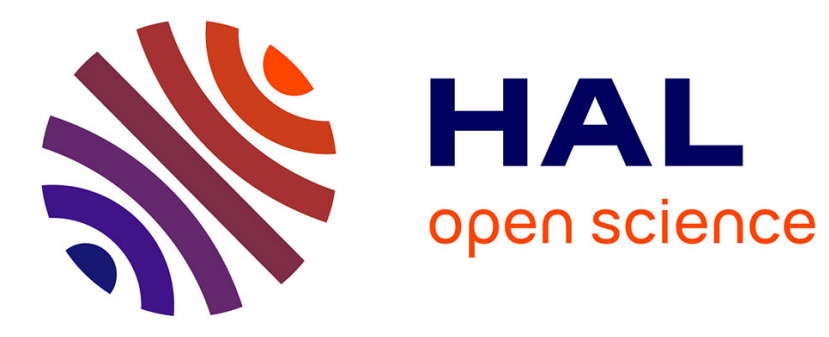

\title{
Spatially Varying Monte Carlo Sure for the Regularization of Biomedical Images
}

Marco Pizzolato, Rachid Deriche, Erick Jorge Canales-Rodriguez, Jean-Philippe Thiran

\section{- To cite this version:}

Marco Pizzolato, Rachid Deriche, Erick Jorge Canales-Rodriguez, Jean-Philippe Thiran. Spatially Varying Monte Carlo Sure for the Regularization of Biomedical Images. ISBI 2019 - IEEE 16th International Symposium on Biomedical Imaging, Apr 2019, Venice, Italy. pp.1639-1642, 10.1109/ISBI.2019.8759338 . hal-02401132

\section{HAL Id: hal-02401132 \\ https://hal.science/hal-02401132}

Submitted on 11 Dec 2019

HAL is a multi-disciplinary open access archive for the deposit and dissemination of scientific research documents, whether they are published or not. The documents may come from teaching and research institutions in France or abroad, or from public or private research centers.
L'archive ouverte pluridisciplinaire HAL, est destinée au dépôt et à la diffusion de documents scientifiques de niveau recherche, publiés ou non, émanant des établissements d'enseignement et de recherche français ou étrangers, des laboratoires publics ou privés. 


\title{
SPATIALLY VARYING MONTE CARLO SURE FOR THE REGULARIZATION OF BIOMEDICAL IMAGES
}

\author{
Marco Pizzolato $^{\star *}$ Rachid Deriche ${ }^{\dagger} \quad$ Erick Jorge Canales-Rodríguez $z^{\ddagger}$ Jean-Philippe Thiran ${ }^{\star \ddagger \diamond}$ \\ ${ }^{\star}$ Signal Processing Lab (LTS5), École Polytechnique Fédérale de Lausanne, Lausanne, Switzerland \\ ${ }^{\dagger}$ Athena Project-Team, Inria Sophia Antipolis-Méditerranée, Université Côte d'Azur, France \\ ${ }^{\ddagger}$ Radiology Department, CHUV, Lausanne, Switzerland, ${ }^{\diamond}$ UNIL, Lausanne, Switzerland
}

\begin{abstract}
Regularization, filtering, and denoising of biomedical images requires the use of appropriate filters and the adoption of efficient regularization criteria. It has been shown that the Stein's Unbiased Risk Estimate (SURE) can be used as a proxy for the mean squared error (MSE), thus giving an effective criterion for choosing the regularization amount as to that minimizing SURE. Often, due to the complexity of the adopted filters and solvers, this proxy must be calculated with a Monte Carlo method. In practical biomedical applications, however, images are affected by spatially-varying noise distributions, which must be taken into account. We propose a modification to the Monte Carlo method, called svSURE, that accounts for the spatial variability of the noise variance, and show that it correctly estimates the MSE in such cases.
\end{abstract}

Index Terms - Stein's Unbiased Risk Estimate (SURE), regularization, anisotropic filter, MRI

\section{INTRODUCTION}

In biomedical imaging, low sensitivity and/or high instrumental noise often requires the use of filters to perform denoising/restoration. In image processing, a plethora of methods has been developed based on different criteria. For instance, when dealing with the human visual perception of images, the quality of the restoration may be assessed as function of the structural information degradation [1]. In other cases, a measure of image content is used [2]. However, biomedical images acquired for instance in Magnetic Resonance Imaging (MRI) are often not ready for human intelligibility but are instead used for quantitative measurements and/or constitute the basis for further processing. As an example, spin-echo images are used to compute relaxation images. In such cases, a measure for the restoration quality should have a more quantitative meaning such as the mean squared error (MSE) of the denoised image with respect to the original noise-free groundtruth one. Obviously, such a metric is not directly accessible in MRI experiments thus it has to be estimated. Stein [3]

\footnotetext{
*Corresponding author: marco.pizzolato@epfl.ch
}

proposed a measure, thereafter named Stein's Unbiased Risk Estimate (SURE), that provides an unbiased estimate of the MSE without having access to the original ground-truth image. Many methods employing this criterion have been devised and are found in the literature. However, the calculation of SURE is not straightforward and an explicit solution is available for linear filters but is generally not accessible for non-linear filters, unless considering very specific cases. To overcome this limitation, Ramani et al. [4] proposed a Monte Carlo calculation of SURE that is suitable for a large variety of filters. Indeed, SURE is based on the knowledge of the noise variance - given or pre-estimated - and of the operator/filter divergence with respect to the data. There is generally no explicit solution for the divergence, so this is approximated through the Monte Carlo calculation. Particularly, the divergence of the operator is estimated by probing the response of the operator itself, in an input-output experiment, with additive Gaussian noise. In this work, to comply with the local nature of the noise affecting biomedical images such as in MRI, we propose to inject information about the noise variance spatial variability when probing the input-output system with Gaussian noise, obtaining the spatially varying SURE (svSURE). We show with simulations that such an adaptation allows for estimating the MSE when regularizing an entire image at once. This allows having an effective sample size, i.e. the number of pixels considered by the operator, that is large enough to avoid fluctuations on repeated estimates, as opposed to local patch-based operators - where the variance could be considered stationary per patch - that would be less suited for Monte Carlo estimation. We point out that, differently from other "weighted" or "adaptive" versions of SURE $[5,6]$ that aim at estimating the weighted MSE or the local optimal filter, svSURE aims at estimating the MSE when the noise is spatially varying in combination with a global spatially varying operator.

\section{IMAGE REGULARIZATION}

We consider a bi-dimensional operator on an image $I_{0}$, defined on $x \in \Omega_{x}$ and $y \in \Omega_{y}$, that admits a well-defined 
second order Taylor expansion. It includes a regularization term implementing some function, $R(I)$, on the image $I$, and an attachment to data term that is weighted by a weighting function, $w(x, y)$, which expresses the amount of confidence in the data, typically proportional to the inverse variance, $w(x, y) \sim 1 / \sigma^{2}(x, y)$. The final operator, $\rho(\cdot)$, is defined as

$$
\begin{aligned}
\rho\left(I_{0}, \lambda\right)= & \inf _{I(x, y)} \lambda \int_{\Omega_{x}, \Omega_{y}} w(x, y)\left[I_{0}(x, y)-I(x, y)\right]^{2} d x d y \\
& +R(I)
\end{aligned}
$$

where the scalar $\lambda$ regulates the compromise between the two terms. In the present work, we implemented a highly non-linear regularization term, following the formulation proposed by Kornprobst et al. [7], that allows for constant smoothing perpendicularly to the local gradient direction while selectively smoothing along it, i.e. across edges, as a function of the gradient norm as $\exp \left(-\|\nabla I\|^{2} / 2 \theta^{2}\right)$, i.e. smoothing across edges is damped in the proximity of an edge with $\theta$ regulating the speed. The operator is evaluated numerically with an in-house implicit step iterative method.

\section{SPATIALLY VARYING SURE}

The Stein's unbiased risk estimate, $\varphi$, for an image $I_{0}$ as a function of $\lambda$ is defined as

$$
\varphi_{I_{0}, \lambda}=\frac{1}{X Y}\left\|I_{0}-\rho\left(I_{0}, \lambda\right)\right\|_{2}^{2}-\sigma^{2}+\frac{2 \sigma^{2}}{X Y} \operatorname{div}\left[\rho\left(I_{0}, \lambda\right)\right]
$$

where $X=\left|\Omega_{x}\right|, Y=\left|\Omega_{y}\right|$, and $\operatorname{div}[\cdot]$ indicates the divergence of the operator that will be estimated with the Monte Carlo method. Indeed, this can be approximated [4] as

$$
\begin{aligned}
& \operatorname{div}[\rho(I, \lambda)] \approx \\
& \quad \frac{1}{\varepsilon} \operatorname{vec}\left[b_{X Y}\right]^{T} \operatorname{vec}\left[\rho\left(I+\varepsilon b_{X Y}, \lambda\right)-\rho(I, \lambda)\right]
\end{aligned}
$$

where $b_{X Y}$ is an image of size $X \times Y$ which entries are drawn from an identically distributed zero mean Gaussian, $N(0,1)$, and where 'vec' indicates vectorization. The approximation is more valid as $\varepsilon \rightarrow 0$, but typically a value $\varepsilon=0.01$ is sufficient. In order to account for the noise spatial variability, while using the operator in eq. (1), we propose to multiply $b_{X Y}$ by the square root of the locally estimated variance normalized such that the total noise energy corresponds to that of unit variance throughout the whole image

$$
b_{X Y}^{s v}(x, y)=b_{X Y}(x, y) \sqrt{\frac{\sigma^{2}(x, y)}{\sum_{x=1}^{X} \sum_{y=1}^{Y} \sigma^{2}(x, y)} X Y}
$$

while respecting the condition of bounded higher order moments [8]. By substituting $b_{X Y}^{s v}$ to $b_{X Y}$ in eq. (3) we can estimate the divergence of an operator that operates differently as a function of the local noise variance, as it is the case of that expressed in eq. (1). This enables de facto the use of SURE with spatially varying Gaussian noise, as we shall show in the following synthetic experiments.

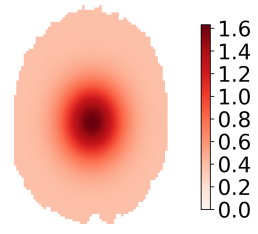

(a) $q=0.5$

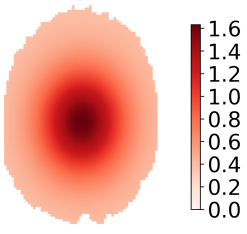

(b) $q=0.7$

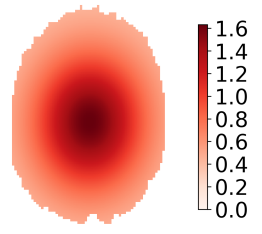

(c) $q=0.9$
Fig. 1: Different tested spatial variability maps.

\section{EXPERIMENTS AND RESULTS}

The proposed svSURE requires the knowledge of the local noise variance. For this reason, we focus our synthetic experiments on MRI images where this information is available through measurements or estimates $[9,10]$. Typical magnitude MRI images are affected by Rician-like noise distribution, but this can be considered Gaussian for signal-to-noise ratios (SNRs) higher than five [11], and eventually unbiasing [12] can be used for the other cases. We simulate T2 relaxation-based Spin-Echo realistic synthetic MRI images with Fiberfox [13], to which we add spatially varying Gaussian noise with different mean $\mathrm{SNR} \in[2,6]$. Spatial variability of the noise variance is simulated according to realistic values as a bivariate Gaussian surface such that the variance is high in the center of the image, decreasing radially towards the borders. Figure 1 shows three examples of the standard deviation spatial variability where the value of $q$ represents a multiplier for the horizontal and vertical standard deviations of the surface which are fixed to 18 and 23 pixels respectively. Figure 2 shows a simulated ground-truth spin-echo image (a) and an example of spatially varying additive noise (b).

For a given SNR and spatial variability map, we calculate the SURE and svSURE estimates of the mean squared error as a function of the regularization parameter $\lambda$, based on the operator in eq. (1) with $\theta=150$. The operation is repeated ten times so that we can obtain a mean value of the estimates and the standard deviation, which are reported in the plots of fig. 3. In the top row we report results for the proposed spa-

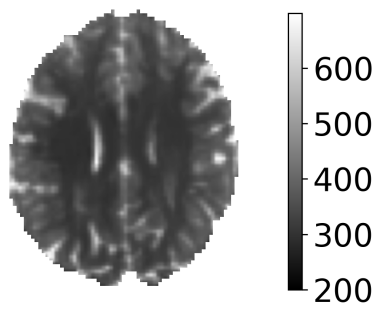

(a) ground-truth

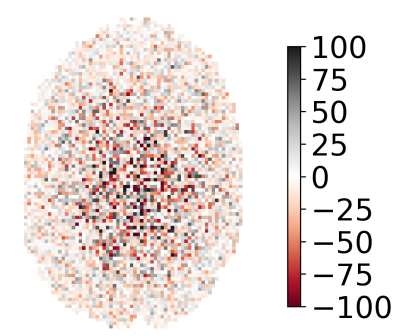

(b) noise ( $\mathrm{SNR}=3, q=0.8$ )
Fig. 2: Synthetic dataset. 

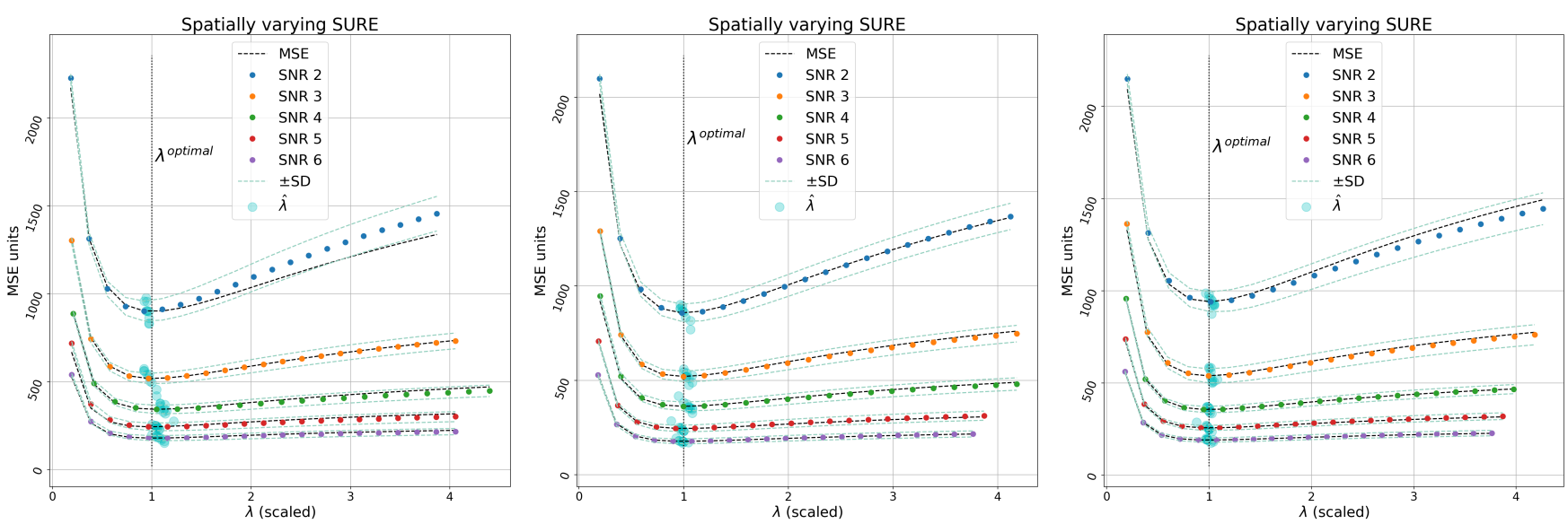

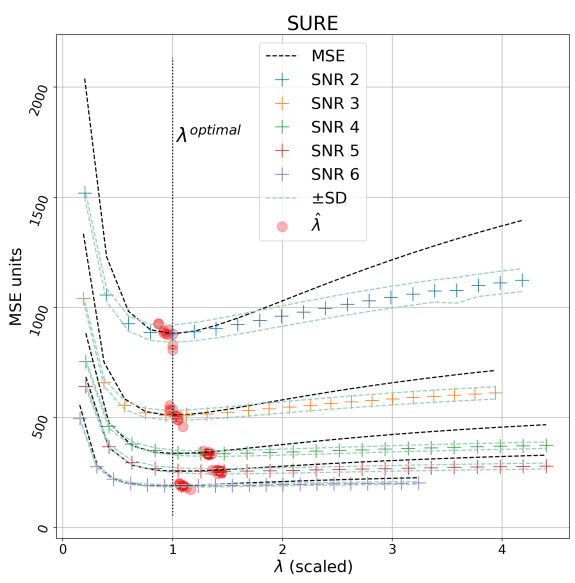

(a) $q=0.5$

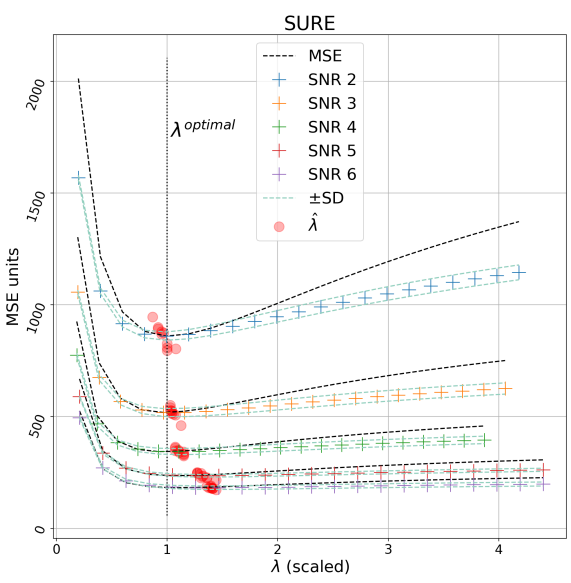

(b) $q=0.7$

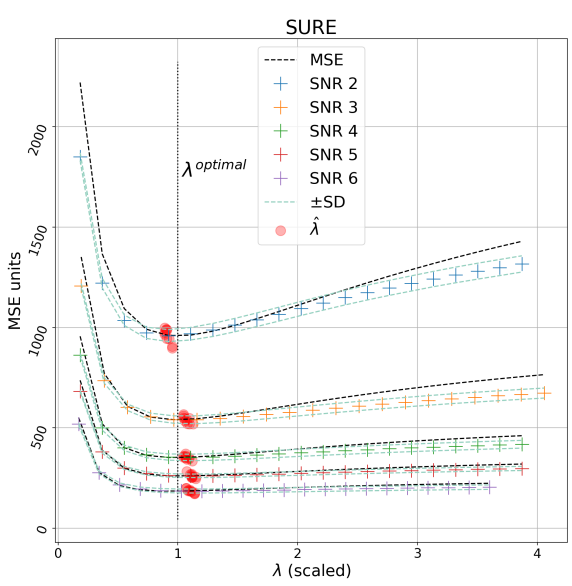

(c) $q=0.9$

Fig. 3: Difference between the proposed svSURE (spatially varying, on the top), and SURE (stationary, bottom), for different SNRs, and for increasing values of $q$, i.e. with noise spatial variability that becomes more homogeneous from left to right.

tially varying svSURE (means indicated by filled circles), and in the bottom row we report results for the stationary version of SURE (means with "+" symbol). In the plots, the values of $\lambda$ for each SNR (encoded by different colors) are different and scaled such that the minimum of the mean squared error (dashed black lines) lies on the vertical dotted line that corresponds to one. With this alignment, it appears clear that the spatially varying svSURE consistently estimates the MSE across the whole range of tested $\lambda$ values, SNRs, and spatial variability maps (each column of the figure is obtained for a different $q$ ). We note that SURE (bottom row) systematically misestimates the MSE not only at low SNR values, but also at higher ones. The misalignment of SURE with the MSE depends on the nature of the spatial variability map. Indeed, as illustrated in fig. 1, as the value of $q$ increases the spatial variability reduces (noise is more homogeneous across the image), thus resembling more and more a stationary case and favouring the overlap. On the contrary, svSURE always performs correctly and guarantees a good overlap with the MSE.

When using a criterion for automatic regularization, the main objective is to identify the optimal amount of regularization (or attachment to data), i.e. to find an estimate $\hat{\lambda}$ that minimizes the mean squared error. In fig. 3 it is possible to visualize all the ten attempts of estimating such a value, individually for each MSE (cyan circles for SVSURE and red ones for SURE). These were obtained by minimizing the two versions of eq. (2), with the divergence estimated with or without using eq. (4), via a golden-section bisection method [14]. Results illustrate that when minimizing the MSE through a proxy such as SURE it is important to consider the spatial variability of the noise as proposed in eq. (4). For instance, in the bottom plots of fig. 3 we note that the estimated values of $\hat{\lambda}$ are biased, whereas the cyan circles in the top plots are always much better aligned on the dotted vertical line corresponding to one, i.e. the optimal $\lambda^{\text {optimal }}$. As expected, the performance of the stationary version decreases with lower values of $q$, i.e. with increasing noise spatial variability.

In order to quantify the performance of the methods, we calculate the relative loss in MSE resulting from a wrong estimation of the minimum, performing none or several repeti- 


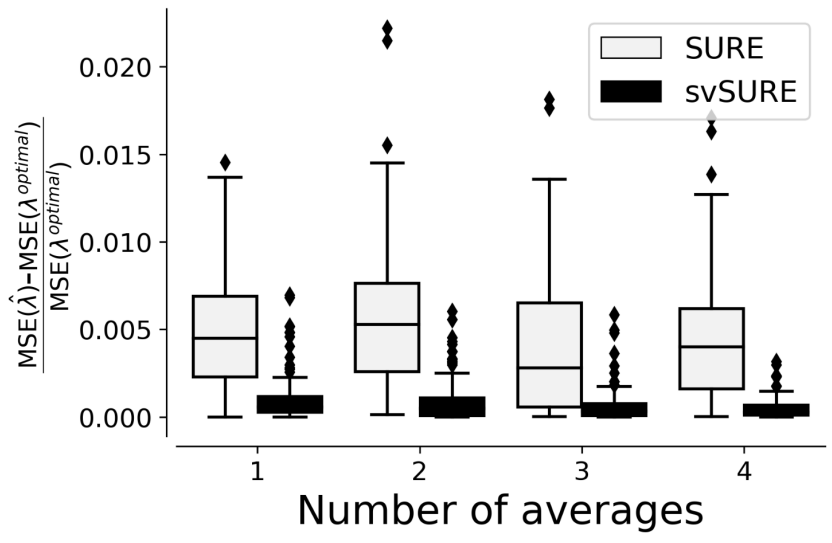

Fig. 4: Relative MSE loss compared to the optimal solution, accounting for a single estimation with different filters, $\theta \in[150,250,350]$, different spatial variability maps, $q \in$ $\{0.5,1\}$, and different signal-to-noise ratios, $\mathrm{SNR} \in\{2,6\}$. The number of averages refers to the number of Monte Carlo iterations used to compute the mean estimate of the MSE.

tions for averaging the estimates of eqs. (3) and (4). The results shown in fig. 4 are computed once but including a large combination of operators as in eq. (1) (values of $\theta$ ), $q$, and SNRs. The stationary version of SURE leads to errors that do not decrease with the number of Monte Carlo repetitions ("averages" in the plot) used to calculate the averaged estimate of the MSE, thus featuring the presence of a systematic bias. Conversely, svSURE visibly entails a negligible loss.

\section{DISCUSSION AND CONCLUSION}

The proposed spatially varying Monte Carlo SURE, svSURE, allows the correct estimation of the mean squared error and its minimum, thus enabling the use of Stein's criterion for the case in which a global spatially varying operator is applied on data corrupted by spatially varying Gaussian noise. We demonstrated this by using different non-linear operators, noise levels, and noise spatial variability maps, from less to more homogeneous. The results suggest that to perform automatic regularization it is fundamental to account for the noise variance spatial variability. The amount of compensated misestimation also depends on the input image, so it is likely that for different scenarios and imaging modalities the need of svSURE could even be more marked.

\section{ACKNOWLEDGMENTS}

Marco Pizzolato is supported by the Swiss National Science Foundation under Sinergia grant number CRSII5_170873. Rachid Deriche is supported by the ERC Advanced Grant agreement No 694665:CoBCoM under the European Union's Horizon 2020 research and innovation program.

\section{REFERENCES}

[1] Wang et al., "Image quality assessment: from error visibility to structural similarity," IEEE Trans. Image Process., vol. 13, no. 4, pp. 600-612, 2004.

[2] Zhu X. and Milanfar P., "Automatic parameter selection for denoising algorithms using a no-reference measure of image content," IEEE Trans. Image Process., vol. 19, no. 12, pp. 3116-3132, 2010.

[3] Stein C.M., "Estimation of the mean of a multivariate normal distribution," The Annals of Statistics, pp. 11351151, 1981.

[4] Ramani et al., "Monte-Carlo SURE: A black-box optimization of regularization parameters for general denoising algorithms," IEEE Trans. Image Process., vol. 17, no. 9, pp. 1540-1554, 2008.

[5] Ramani et al., "Non-cartesian MRI reconstruction with automatic regularization via Monte-Carlo SURE," IEEE Trans. Med. Imaging, vol. 32, no. 8, pp. 1411-1422, 2013.

[6] Li et al., "SURE-based optimization for adaptive sampling and reconstruction," ACM Trans. Graphics, vol. 31, no. 6, pp. 194, 2012.

[7] Kornprobst et al., "Non-linear operators in image restoration," in CVPR. IEEE, 1997, p. 325.

[8] Hutchinson M.F., "A stochastic estimator of the trace of the influence matrix for laplacian smoothing splines," Communications in Stat. Sim. Comp., vol. 19, no. 2, pp. 433-450, 1990.

[9] Manjón et al., "MRI noise estimation and denoising using non-local PCA," Med. Image Anal., vol. 22, no. 1, pp. 35-47, 2015.

[10] Pieciak et al., "Non-stationary rician noise estimation in parallel MRI using a single image: a variancestabilizing approach," IEEE Trans. Pattern Anal. Mach. Intell., vol. 39, no. 10, pp. 2015-2029, 2017.

[11] Pajevic S. and Basser P.J., "Parametric and nonparametric statistical analysis of DT-MRI data," Journal of magnetic resonance, vol. 161, no. 1, pp. 1-14, 2003.

[12] Kaden et al., "Quantitative mapping of the per-axon diffusion coefficients in brain white matter," Magn. Reson. Med., vol. 75, no. 4, pp. 1752-1763, 2016.

[13] Neher et al., "Fiberfox: facilitating the creation of realistic white matter software phantoms," Magn. Reson. Med., vol. 72, no. 5, pp. 1460-1470, 2014.

[14] Braun W.J. and Murdoch D.J., A first course in statistical programming with $R$, Cambridge Univ. Press, 2007. 